\title{
ALTERNATIVAS ÉTICAS DESDE LA EDUCACION AMBIENTAL
}

ETHICAL ALTERNATIVES FROM ENVIRONMENTAL EDUCATION

ALTERNATIVAS ÉTICAS DA EDUCAÇÃO AMBIENTAL

Manuel Villarruel-Fuentes*

(iD) https://orcid.org/oooo-0002-1174-0528

María de Lourdes Villarruel-López ${ }^{* *}$

(iD) https://orcid.org/oooo-0oo2-3987-966o

Resumen: En medio de intensas contiendas ideológicas por ganar los espacios de entendimiento en torno al destino que habrá de enfrentar la humanidad, las propuestas éticas cobran una gran vigencia, constituyéndose en referentes del actuar correcto en tiempos de crisis. Con base en ello, la educación ambiental brinda el sustrato necesario para recuperar los valores y principios que permiten repensar la conducta humana respecto a los distintos contextos de actuación social, política, económica y cultural-, así como reinstalar la relación armónica que antaño se tuvo con el ambiente natural. Como producto de estas disertaciones se trazan rutas de pensamiento reflexivo y crítico y se discuten propuestas desde los marcos que definen el saber, los valores y las creencias como base sustantiva de una ciudadanía ambiental y de una ética para la vida.

Palabras clave: Educación superior; ambiente natural; ciudadanía ambiental.

Abstract: In the midst of intense ideological struggles to win the spaces of understanding around the destiny that humanity will have to face, the ethical proposals gain a great validity, constituting themselves as references of the correct act in times of crisis. On this basis, environmental education provides the necessary substrate to recover the values and principles that allow rethinking human behavior, respect for different contexts of action -social, political, economic and cultural-, as well as reinstating the harmonious relationship had with the natural environment. As a result of these dissertations, reflective and critical thinking paths are traced and proposals are discussed from the frames that define knowledge, values and beliefs as the substantive basis of an environmental citizenship and an ethic for life.

Keyword: Higher education; natural environment; environmental citizenship.

Resumo: Em meio a intensa luta ideológica para ganhar os espaços de entendimento em torno do destino que a humanidade enfrentará, as propostas éticas entram em vigor, tornando-se referentes da ação correta em tempos de crise. Com base nisso, a educação ambiental proporciona o substrato necessário para recuperar os valores e princípios que permitem repensar o comportamento humano em relação aos diferentes contextos de ação - social, político, econômico e cultural - bem como restabelecer a relação harmônica com o meio ambiente natural. Como resultado dessas dissertações, traçam-se rotas reflexivas e críticas de pensamento e discutem-se propostas a partir dos referenciais que definem conhecimentos, valores e crenças como base substantiva de uma cidadania ambiental e de uma ética para a vida.

Palavras chave. Educação superior; ambiente natural; cidadania ambiental. 


\section{Introducción}

Los nuevos contextos de actuación docente, dentro de la educación superior en América Latina, se significan no solo por el constante cambio en las formas y los medios de operar las propuestas curriculares y didácticas inmersas en los planes y programas de estudio, sino también por su acentuada decantación hacia los modelos pragmáticos y utilitarios, centrados en el desarrollo de la experticia y el adiestramiento situado, tales como los derivados del enfoque de competencias profesionales (GARCÍARETANA, 2011; LEÓN-URQUIJO, RISCO DEL VALLE, Y ALARCÓN SALVO, 2014; ZAPATA-CALLEJAS, 2015). Al respecto, los procesos de intervención educativa mediados por las Tecnologías de la Información y la Comunicación (TIC), se han constituido en el soporte toral donde descansan las expectativas de logro académico, tanto de maestros como de estudiantes. Sobre este particular se destaca como:

La expectativa benéfica de las TIC en el sistema educativo y las condiciones en las que dicha expectativa se hace posible ponen en evidencia la necesidad de realizar cambios en todas sus áreas (técnica, pedagógica, administrativa, directiva), para que de esta manera se puedan suscitar experiencias educativas eficaces y efectivas que favorezcan los procesos de enseñanza y aprendizaje. (VALENCIA-MOLINA et al., 2016, p.8).

$\mathrm{Si}$ bien esto pone en relieve una estrategia ampliamente documentada y discutida dentro del campo de la investigación educativa, lo cierto es que se pasa por alto la necesidad de generar un cambio en las relaciones interpersonales, a partir de modificar las actitudes y aptitudes de los protagonistas del hecho educativo. En este sentido, ninguna reforma en las directrices operativas dentro de las instituciones de educación superior podrá, por sí misma, alcanzar los objetivos señalados. El problema no solo es técnico o administrativo, lo es también humano.

En conceptos de Torres-Rivera et al., (2014) el abordaje de la investigación vinculado a la educación superior "se ha centrado en el análisis de la naturaleza de la educación bajo el enfoque de las competencias [...], pero no en la naturaleza de la práctica docente y sus competencias como profesional de la educación...” (p.13o). Se trata de un esquema fundado desde el ideario de una economía del conocimiento, donde los saberes se constituyen en activos de los grupos sociales, particularmente de las empresas.
* Doctor en Educación. Profesor Investigador en el Tecnológico Nacional de México/Campus Úrsulo Galván. Líder del Cuerpo Académico "Cultura Académica y Desarrollo Social Sustentable" y de la Línea de Investigación "Docencia y Aprendizaje". Presidente de la Red Veracruzana de Investigación e Innovación Educativa. Miembro del Consejo Mexicano de Investigación Educativa.

E-mail: dr.villarruel.fuentes@gmail.com.

** Maestra en Investigación Educativa Egresada de la Universidad Veracruzana. Doctoranda en Pedagogía por la Universidad Nacional Autónoma de México. Docente de Grado y Posgrado en el Nivel Superior Universitario y Consultora Experta en Educación Ambiental.

E-mail: lulu.villarruel.lo@gmail.com. 
Desde estas instancias conceptuales y operativas se cosifica al ser humano, concibiéndolo como depositario de las habilidades necesarias para operar en ambientes laborales, y no necesariamente en los entornos socioculturales.

Este aparente sesgo no ha sido producto de una desatención, sino del sentido que se le ha dado a la educación basada en competencias, entendida como la imbricación de lo conceptual, lo axiológico y lo procedimental, expresado en un proceso virtuoso que se identifica de facto con la formación integral. Desde esta perspectiva, es posible observar la configuración de una estructura cultural monolítica, una especie de tótem social, desde el cual se proyecta una serie de principios legaliformes, condensados en una ética única e indiscutible, que justifica el actual apego a lo material. Esto explica por qué la afiliación a la misión y visión propias de los consorcios comercialempresariales, ha permeado tan profundo en el tejido social en América Latina, al grado de que incluso las instituciones de educación superior han tenido que alinearse con este pensamiento dominante, que dicta cánones de desempeño a partir del nivel de competencias adquiridas. En conceptos de Avilla-Colla (2014), los seres humanos buscan producir por la fuerza algo que no pueden encontrar en la naturaleza, un orden antes impensable y, sin embargo, en apariencia necesario para el bienestar social deseado.

De esta forma, los fundamentos éticos necesarios para la vida social y el desarrollo cultural han quedado circunscritos a simples slogans publicitarios, desde donde se concibe y proyecta la nueva naturaleza humana, sus relaciones y aspiraciones deéxito. Setrata de una orientación que sobrevalora el impacto de las destrezas adquirida por las personas, pero a su vez, reduce a su mínima expresión el componente humano, al recluir los valores y principios en una ética que lo explica todo, para todos y en cualquier contexto. Álvarez-Pérez, González-Alonso y López-Aguilar (2009) lo aclaran al señalar que las "competencias amplias, gestión de conocimientos, competencias para el manejo de la información, competencias de empleabilidad aplicables a distintas situaciones y contextos son los nuevos mensajes y los nuevos conceptos a los que la institución universitaria debe estar atenta" (p.3).

Una pregunta es obligada: ¿dónde queda la dimensión ética dentro de estas propuestas educativas? Al parecer no existe intención de definirla, menos aún de incorporándola dentro de los debates educativos actuales. Por ello es preciso la construcción de un basamento conceptual que permita recuperar la reflexión sobre sus posibilidades de inclusión dentro de las propuestas curriculares y didácticas, a fin de no renunciar al crecimiento humano, al fomento de su talento y creatividad, pero también de su sensibilidad, 
para desde ahí construir nuevas formas de relacionarse con la naturaleza. El objetivo es buscar nuevas formas de entender lo social, de comprender y abordar el nuevo ethos educativo. Aquí es donde se abre la posibilidad de considerar a la educación ambiental como el medio para lograr un cambio sustancial en las conductas éticas de los estudiantes, en virtud de ser depositaria de un sólido modelo de valores, auspiciados desde las vertientes más humanistas del crecimiento humano, donde el ambiente es entendido como:

...el espacio biofísico y social en el cual vivimos. Nuestro espacio de vida y de cultura. No se trata sólo de la naturaleza (plantas y animales) sino que incluye también al ser humano y su actividad cultural. Ahora bien, cuando se habla de la ética ambiental, de esta reflexión sobre nuestras acciones en relación con el ambiente, pues, al igual que para la ética en general, también existe aquí una diversidad de perspectivas acerca de lo que son las buenas o malas acciones sobre el ambiente. (OLAGUEZ-TORRES y ESPINOROMAN, 2012, p. 2181).

Se aprecia en principio la necesidad de "hablar de ambiente y no de medio ambiente, entendido este último sólo como el entorno físico que nos influye y soporta la carga de la sociedad humana" (ALVINO, CANCIANI, SESSANO y TELIAS, 2007, p.153).

Se trata, tal como afirma González-Escobar (2017), de entender el decisivo papel de la educación ambiental, sobre todo en el desarrollo de una formación crítica en los seres humanos, asumiéndolos como sujetos que comprenden la complejidad y multidimensionalidad de los problemas ambientales, así como la crisis planetaria que le distingue. Desde sus conceptos, la educación ambiental es el campo emergente desde el que se puede enfrentar los desafíos de una formación integral y ética: el desarrollo humanista, la preparación científica y el pensar transdisciplinar, reconociendo a los sujetos como seres cognitivos y políticos, que pueden y deben asumir un compromiso con la sostenibilidad de sus territorios. Todo ello en busca de "conseguir sociedades más armónicas y equitativas" (NOVO, 2009, p. 198).

Es por demás evidente que la sociedad ha cambiado, y nosiempreparamejorar.Enlosúltimos40añosla "economía se volvió economía ecológica, la ecología se convirtió en ecología política, y la diversidad cultural condujo a una política de la diferencia" (GALANO; CURI; MOTOMURA; PORTO-GONCALVES; SILVA; ÁNGEL, et al., 2002, p. 2), pero los cambios sustanciales esperados no terminan 
de llegar. En conceptos de estos autores la ética está transmutando a una ética política. Todo parece indicar que, de seguir este rumbo, la polarización existente entre las utopías educativas y las razones prácticas del mercado, aunado a las diferencias entre los intereses creados y los valores socialmente necesarios, conducirán hacia una economía moral, ligada al "mayor logro silencioso neoliberal: una transformación cultural que convirtió la riqueza monetaria personal en el valor (en sentido axiológico) supremo y el criterio económico en el único válido en las decisiones públicas" (BOLTVINIK, 2019, s/p).

Sobre esta base argumentativa en el presente artículo se plantea la posibilidad de gestionar un andamiaje conceptual para pensar una nueva ética desde las dimensiones de la actual crisis planetaria, cuyo epicentro se configure a partir de los fundamentos epistémicos de la educación ambiental, y más específicamente de aquella que se concibe para la sustentabilidad.

\section{Desarrollo}

Las actuales propuestas educativas en América Latina han comprometido sus esfuerzos en la búsqueda y consolidación de un proceso innovador que les permita alinearse con los contextos internacionales, sustentadas, como ya se señaló, en el denominado enfoque por competencias; una perspectiva que en palabras de Escudero (2008) está emparentada con el conocido modelo por objetivos, en el cual se fijan los estándares de aprendizaje para los titulados y se minimizan los contenidos a un sentido instrumental y utilitarista. Es decir, se mantienen viejos modelos mecanicistas disfrazados de aparentes enfoques innovadores.

Bajo esta vertiente de pensamiento, se han privilegiado ciertos saberes, valores y prácticas, evidentemente supeditadas a escenarios socioeducativos monolíticos, donde impera la razón lógica de unos cuántos sobre los intereses de la mayoría. Situación que se ve reflejada en una sociedad consumista, extraviada en los valores esenciales, catalogados como indispensables para la convivencia humana, lo que condiciona una severa crisis civilizatoria -de origen axiológico- que compromete el porvenir y la vida en el planeta.

Ante dicho panorama, se requiere recuperar el sentido que se le ha dado a la educación y arribar a senderos más críticos, reflexivos y holísticos que permitan comprender y actuar en «el todo», ponderándolo por encima de sus partes, donde no se enaltezca el homogeneizar la conciencia ciudadana en aras de mantener las antiguas prácticas que 
han llevado al deterioro del tejido social y ambiental, a la crisis de valores y al menosprecio de lo natural.

Por tal motivo se hace pertinente recuperar a la Educación Ambiental (EA) como generadora y promotora del cambio sustancial que se reclama, posible desde la génesis de una renovada relación entre el ser humano y la naturaleza, vínculo concebido como el espacio ideológicoconceptual donde fluyen las contradicciones y emanan las pugnas y los sinsentidos.

Sin embargo, los matices teóricos de la EA son amplios y derivan de las distintas concepciones y relaciones que se han generado entre el ser humano y el ambiente natural. Manifestaciones construidas desde los distintos enfoques que se tienen de este último: algunos conservacionistas, otros utilitaristas, identitarios o sistémicos, otros más resolutivos -colaborativos y críticos-, tal como lo refiere en su tipología Sauvé (2004), los cuales reflejan el significado que asume el ambiente para la sociedad, configurado a partir de la capacidad transformadora que se tiene sobre él, así como la valoración inclusiva o exclusiva que se le otorga al ambiente en la dinámica social, o bien, concebido como un componente ajeno a ella, sin descartar las posibilidades que se le confiere como modelador y guía del pensamiento y los valores humanos implicados.

Resulta relevante entender cómo los fundamentos de estos enfoques, fundamentados en torno a la ontología del ser han permeado y mediado su relación con la naturaleza, ya que a partir de la manera de concebirla es la forma en que se interactúa con ella y, a su vez, las interacciones transforman las concepciones que se tienen en torno a la misma.

De esta manera, ante los distintos posicionamientos, "la discusión ética en el campo del Ambientalismo nos remite directamente a la discusión sobre el antropocentrismo y el biocentrismo" (GONZÁLEZ-GAUDIANO y FIGUEROA, 2009, p.104). Dos corrientes propias de la ética ambiental que entre sus proyecciones priorizan uno de los disensos más representativos de toda discusión: la naturaleza o el ser humano, tal como se observa en la propuesta de Foladori (2005).

Se trata de un debate filosófico que a decir de Lecaros-Urzúa (2013) se ha centrado en tres líneas: (i) el diagnóstico sobre las causas históricas, sociales, culturales, políticas, jurídicas y económicas de la crisis socioecológica en un nivel planetario; (ii) la atención en los intereses morales de las generaciones futuras de seres humanos y (iii) la presunción del valor otorgado, sobre la que se basan las obligaciones ético-ecológicas, fragmentadas en valores instrumentales, extrínsecos a la naturaleza y 
fundados en los intereses humanos -que cimientan los antropocentrismos éticos-, y en valores intrínsecos a la naturaleza o a los seres vivos que la integran, fundamento de los biocentrismos y ecocentrismos en ética ambiental.

Ante dicha disyuntiva, Foladori (2005) señala que el biocentrismo o ecocentrismo prioriza la esfera natural que impone ciertos comportamientos a la sociedad, dotando a la naturaleza de valores intrínsecos que son definidos por los mismos seres humanos, quienes usufructúan espacios naturales, erigiéndose como consumidores. Por otro lado, el autor señala que es en el antropocentrismo donde el interés humano tutela el criterio de valor inmerso en la relación ambiente/sociedad, y distingue entre tecnocentristas -quienes consideran que la tecnología y sus procesos podrán solucionar problemas ambientales específicos- y los marxistas -identificados también como clasistas o humanistas-, que no diferencian entre lo artificial y lo natural, al no percibir una separación entre la naturaleza y la sociedad; estiman que la sociedad está integrada por grupos y clases sociales, con intereses contrapuestos, cuyas contradicciones sociales explican el comportamiento humano respecto a la naturaleza.

Dentro del campo de la sustentabilidad, el biocentrismo y el antropocentrismo han sido equiparados con la sustentabilidad fuerte y la sustentabilidad débil (o desarrollo sustentable) respectivamente, tal como lo señala Pierri (2001), citado por González-Gaudiano y Figueroa (2009). Sin embargo, se tiene que considerar que el biocentrismo se vincula más con la sustentabilidad superfuerte desarrollada teóricamente por Gudynas (2011). Dentro de este posicionamiento la naturaleza es concebida como un patrimonio natural y el crecimiento es sinónimo de calidad de vida y soporte ecológico, al tiempo que se reconocen múltiples valores humanos, así como los principios intrínsecos a ellos; se habla de justicia ambiental y ecológica, además de ciudadanos y sociedades donde convergen y se priorizan múltiples conocimientos y saberes (GUDYNAS, 2011). En relación a ello, el propio autor enfatiza en que la sustentabilidad débil es antropocéntrica, al homologar al desarrollo con el crecimiento material, donde la naturaleza es referida como capital natural. Se busca además compensar económicamente por las repercusiones ambientales que puedan derivarse de su aplicación, reconociéndose sólo el conocimiento científico por encima de otros saberes, los cuales son ignorados. En este entorno, se habla de consumidores y no de ciudadanos, en un contexto mediado por el mercado; es decir, hay una valoración funcional de la naturaleza, que parte de su concepción epistémica ligada al positivismo. Corriente ideológica propia del colonialismo 
europeo, que a decir de De Souza - Santos (2010) provocó la destrucción de los saberes tradicionales de los pueblos primeros - epistemicidio -, que todavía se percibe vigente, ya que: "el fin del colonialismo político no significó el fin del colonialismo en las mentalidades y subjetividades, en la cultura y en la epistemología y que por el contrario continuó reproduciéndose de modo endógeno" (DE SOUZA- SANTOS, 2010, p.8).

Para el caso de la sustentabilidad fuerte, su postura antropocéntrica concibe al desarrollo como crecimiento material, vinculado al bienestar de la sociedad, donde se prioriza el conocimiento científico y se minimizan los demás saberes- exaltándose la justicia ambiental y la valoración ecológica con un sentido tecnológico-, la naturaleza es concebida como capital natural, exenta de un enfoque crítico, cosificando la figura de los actores al pensarlos como consumidores y ciudadanos dentro de una sociedad donde prioriza el mercado (GUDYNAS, 2011).

Ante dicho panorama, recuperar los saberes y valores éticos ambientalistas y sustentables puede derivar en distintos discursos y prácticas socialmente dominantes. En este caso el interés no se centra en recuperar los discursos hegemónicos del desarrollo sustentable, sino en acentuar la base axiológica de la Educación Ambiental frente a un sistema educativo que busca adoptar, sin reflexión y sin considerar las necesidades contextuales específicas, toda propuesta educativa. Al respecto, González-Gaudiano y Figueroa (2009) resaltan la necesidad de fomentar valores ambientales en la educación formal, lo cual conlleva no sólo la adquisición de conocimientos, sino la toma de conciencia, de actitudes y conductas que permitan transitar hacia posicionamientos más críticos. A pesar de ello, los autores resaltan que este enfoque, propio de la Educación Ambiental, no tendría que ser impuesto, ya que lo que se busca es una mayor apertura, que privilegie al razonamiento reflexivo y fortalezca la capacidad de las personas para comprometerse con su entorno, por lo que:

Educar en valores, éticamente, es promover en los sujetos el despliegue de una racionalidad moral sustantiva, de modo autónomo y comprometido para adquirir la capacidad de decidir en aquellos asuntos que afecten sus propias vidas con pleno ejercicio de su libertad responsable. (GONZÁLEZGAUDIANO y FIGUEROA, 2009, p.109).

En este sentido y desde las propuestas teóricas ya mencionadas, se busca la construcción de ciudadanos ambientales, que deben promoverse e integrarse desde las entrañas de los propios sistemas educativos, situación 
que será posible en la medida en que se fomente la implementación de enfoques educativos interdisciplinarios, así como la convergencia de diversos saberes, que al mismo tiempo se acompañen de currículos más flexibles y ambientalizados (GONZÁLEZ-GAUDIANO y FIGUEROA, 2009; RODRÍGUEZ, 2011). Pero ello resuelve el qué hacer, pero no aclara el cómo hacerlo. Sobre el particular CastroCuéllar, Cruz- Burguete y Ruiz-Montoya (2009), brindan una propuesta basada en las siguientes interrogantes: ¿Qué hacer para tomar conciencia del respeto que merece la naturaleza? ¿Cómo fomentar valores que fueron parte de antiguas civilizaciones donde prevalecía el respeto y admiración a los elementos naturales? (p.355). Su postura se ubica en:

[...] el reconocimiento más profundo de la codependencia con la naturaleza, el respeto a la vida en cualquiera de sus manifestaciones, la admiración por la generosidad de la Tierra, la benevolencia de plantas y animales, la fascinación por la diversidad ecológica y la humildad ante la majestuosidad de los paisajes y recursos naturales. (CASTROCUÉLLAR, CRUZ- BURGUETE y RUIZMONTOYA, 2009, p. 355).

Actitudes propias de un ser humano ecoalfabetizado, orientado desde un enfoque ecosocial o biocultural que integra el bienestar social con la conservación de la diversidad biológica y cultural (CASTRO-CUÉLLAR, CRUZ- BURGUETE y RUIZMONTOYA, 2009).

Sin embargo Rodríguez (2011), frente al imperante modelo por competencias y la necesidad de fomentar una educación ambiental para la formación de ciudadanos ambientales, proyecta al respecto: “¿Cómo articular y promover las competencias de la Educación Ambiental con las de formación ciudadana conducentes al fortalecimiento de un pensamiento ambiental en la escuela, hacia la construcción de una ciudadanía ambiental?” (p. 13) Pregunta por demás vigente en la actualidad que, en primer lugar, lleva a preguntarse si el modelo por competencias permite la formación de ciudadanos con estas características, constituyéndose en un impedimento para la construcción de la anhelada ciudadanía ambiental.

Pero más allá de ello, la idea de una ética planetaria lleva implícita la impronta del cambio social, cuyo propósito es recuperar los tradicionales idearios culturales, rescatando el sentido de identidad territorial que desde siempre otorgó pertinencia e 
identidad a los modos y las formas de relacionarse con la naturaleza. Desde aquí es posible aspirar al logro de una conciencia universal que pueda nuevamente nutrirse de la unidad planetaria, que respete la biodiversidad en todas sus manifestaciones - ecológica, cultural, política, económica y social-, donde el ser humano nuevamente aspire con voluntad a ser y vivir en comunidad. Ética que en palabras de Morin (1999) debe estar inmersa en la educación del futuro, consagrada a enseñar la condición humana y ubicarla en el mundo.

\section{Conclusiones}

Hablar de ética es rememorar la conducta humana a través de los tiempos. Es un metarrelato que pone en relieve los valores y principios que subyacen a su concreción, es el ethos que identifica y define lo que para las sociedades es bueno y malo. Es de muchas formas una organización de los saberes y experiencias propias y compartidas.

Pero la ética también debe ser vista como una filosofía de la vida, donde a decir de Leff (2006) debe apuntar al deseo de vida, pretensión que admite a la sustentabilidad como la marca del límite de la vida. En sus conceptos, es precisamente la muerte entrópica del planeta la que orienta "la búsqueda de las raíces de la vida, a la voluntad de vida, más allá de la necesidad de conservación de la biodiversidad y del principio de supervivencia de la especie humana" (LEFF, 2006, p.13).

Hoy el mundo enfrenta el reto de pensarse como una gran comunidad global, cuyo sentido de pertenencia se encuentra fragmentado en su orientación teleológica. Las metas, antaño tan diversificadas, han cristalizado en un objetivo común, acorraladas por una matriz semántica que define todo a partir de un código lingüístico monotemático, cargado de pragmatismoy utilidad. Ya las cosas no significan lo que cada comunidad o grupo social desee. Incluso los simbolismos han perdido su polivalencia, lo intangible se ha materializado para ser valorado como objeto físico y las verdades han sido canceladas en su definición. En medio de este contexto ¿QQué queda por hacer?

Ensimismadas en complejas y variadas utopías que proponen el cambio social y cultural, las sociedades actuales deben hacer el último esfuerzo por recuperar su hábitat natural, a cuya transformación se consagró con vehemencia en los dos últimos siglos. Para ello debe comprometer su conciencia y voluntad; dimensiones consustanciales que la humanidad perdió en el proceso civilizatorio.

Si como algunos investigadores e intelectuales proclaman, el verdadero problema es de saberes, 
entonces es fácil advertir cómo la solución anida en un cambio de pensamiento. Entender claramente algo, de alguna manera anula el disenso y la confrontación ideológica. Pero esto va más allá. Mucho del éxito posible en la constitución de una nueva ética, radica precisamente en que no necesariamente debe ser nueva, esto es, las respuestas pueden estar en el pasado, en las viejas tradiciones griegas, en las cosmovisiones de los pueblos primeros, en la cosmogonía espiritual que en el pasado dio origen a las explicaciones del universo, ideario que brindó entendimiento sobre los propósitos de la naturaleza y la función del ser humano en el ciclo de la vida.

Saber, valorar y creer. He aquí la tríada mítica que hace posible las alternativas éticas de la educación ambiental, la existencia de un código de ética sustentado en principios y no en normas. Sin ellos, solo queda la mirada inerte que la ciencia decimonónica instituyó.

\section{Referências}

ÁLVAREZ-PÉREZ, P. R., GONZÁLEZ-AFONSO, M. C. \& LÓPEZ-AGUILAR, D. La enseñanza universitaria y la formación para el trabajo: Un análisis desde la opinión de los estudiantes. Paradígma, v. 30, n. 2, p.7-20. 2009. Recuperado de http://www.scielo. org.ve/scielo.php?script $=$ sci_arttext\&pid $=$ S101122512009000200002\&lng=es\&tlng=es.

ALVINO, S., CANCIANI, L., SESSANO, P. y TELIAS, A. La ciudadanía y el derecho al ambiente: reflexiones en torno a una articulación. Anales de la educación común. Tercer siglo, v.3, n.8, p.152-161. 2007. Recuperado de: http://servicios.abc.gov.ar/lainstitucion/ revistacomponents/revista/archivos/anales/numeroo8/ archivosparadescargar/19_ciudadania.pdf.

AVILLA-COLLA, R. A lógica da organização forçada e as improbabilidades do futuro. Revbea, São Paulo, v. 9, n. 1, p.10-22, 2014. Recuperado de: http://revbea. emnuvens.com.br/revbea/article/view/2311/2830.

BOLTVINIK, J. Economía moral. Sección Opinión, La Jornada. México. 15 de febrero, 2019. Recuperado de: https://www.jornada.com.mx/2019/o2/15/opinion/ o2001eco\#.

CASTRO-CUÉLLAR, A., CRUZ- BURGUETE, J.L. y RUIZMONTOYA, L. Educar con ética y valores ambientales para conservar la naturaleza. Convergencia. Revista de 
Ciencias Sociales. n. 50, p. 353-382, 2009. Recuperado de: http://www.scielo.org.mx/pdf/conver/v16n5o/ v16n50a14.pdf.

DE SOUZA-SANTOS, B. Descolonizar el saber, reinventar el poder. Montevideo: Ediciones Trilce. 2010. Recuperado de: http://www.boaventuradesousasantos. pt/media/Descolonizar\%20el\%20saber_final\%20-\%20 $\mathrm{C} \% \mathrm{C}_{3} \%$ B3pia.pdf.

ESCUDERO-MUÑOZ, J.M.. Las competencias profesionales y la formación universitaria: posibilidades y riesgos. Revista de Docencia Universitaria, v.1, p. 1-20, 2008. Recuperado de https://dialnet.unirioja.es/ servlet/articulo? codigo $=2749780$.

FOLADORI, G. Una tipología del pensamiento ambientalista, en FOLADORI, G. y PIERRI, N., ¿Sustentabilidad? Desacuerdos sobre el desarrollo sustentable. México, Miguel Ángel Porrúa. 2005.

GALANO, C., CURI., M., MOTOMURA, O., PORTOGONCALVES, C. W., SILVA, M.y ÁNGELA. et al. Manifiesto por la vida. Por una ética para la Sustentabilidad.Ambiente \& Sociedade, v.5, n.10, p.1-14. 2002. Recuperado de: http://www.scielo.br/pdf/asoc/n10/16893.pdf.

GARCÍA-RETANA, J. A. Modelo educativo basado en competencias: importancia y necesidad. Revista Electrónica Actualidades Investigativas en Educación, v.11, n.3, p.1-24. 2011. Recuperado de: https://www.redalyc.org/pdf/447/44722178014.pdf.

GONZÁLEZ-ESCOBAR, C. H. La educación ambiental ante el problema ético del desarrollo. Revista Electrónica Educare (Educare Electronic Journal), v. 21, n. 2, p.1-19. 2017. Recuperado de: file://C:/Users/Usuario1/ Downloads/Dialnet-LaEducacionAmbientalAnteElProble maEticoDelDesarrol-5919101.pdf.

GONZÁLEZ-GAUDIANO, E. y FIGUEROA DE KATRA, L. Los valores ambientales en los procesos educativos: realidades y desafíos. Revista Iberoamericana sobre Calidad, Eficacia y Cambio en Educación, v. 7, n. 2, p. 95-115. 2009. Recuperado de https://repositorio. uam.es/bitstream/handle/10486/661169/REICE_7_2_6. pdf? sequence $=1$ \&isAllowed $=\mathrm{y}$.

GUDYNAS, E. Desarrollo y sustentabilidad ambiental: diversidad de posturas, tensiones persistentes. En A. MATARÁN RUÍZ, A; LÓPEZ CASTELLANO, F. (Eds). La Tierra no es muda: diálogos 
entre el desarrollo sostenible y el postdesarrollo, Granada: Universidad de Granada, p. 69-96. 2011. Recuperado de http://gudynas.com/publicaciones/ GudynasUsosIdeasSustentabilidadGranada11.pdf.

LECAROS-URZÚA, J. A. La ética medio ambiental: principios y valores para una ciudadanía responsable en la sociedad global. Acta Bioethica, v.19, n.2, p.177188. 2013. Recuperado de: https://scielo.conicyt.cl/pdf/ abioeth/v19n2/arto2.pdf.

LEFF, E. Ética por la Vida. Polis, n.13, p.1-18. 2006. Recuperado de: http://journals.openedition.org/polis/5354

LEÓN-URQUIJO,A.P., RISCODELVALLE,E.yALARCÓNSALVO, C. Estrategias de aprendizaje en educación superior en un modelo curricular por competencias. Revista de la Educación Superior, v. 43, n. 172, p. 123-144. 2014. Recuperado de: http://publicaciones.anuies.mx/pdfs/ revista/Revista172_S3A6ES.pdf.

MORIN, E. Los siete saberes necesarios a la educación del futuro. Organización de las Naciones Unidas para la Educación, la Ciencia y la Cultura: Francia. 1999. Recuperado de: http://users.df.uba.ar/solari/ Docencia/Complejos/morin.pdf.

NOVO, M. La educación ambiental, una genuina educación para el desarrollo sostenible. Revista de Educación, número extraordinario. p. 195-217. 2009. Recuperado de: http://www.revistaeducacion.mec.es/re2009/re2009 _o9.pdf.

OLAGUEZ-TORRES, J. E. y ESPINO-ROMÁN, P. Ética y valores en educación ambiental. Congreso internacional de investigación. Academia Journals, v.4, n3, p. 21792182. 2012. Recuperado de: file://C:/Users/Usuario1/ Downloads/Tomo15-CELAYA.pdf.

RODRÍGUEZ-PINZÓN, M.C. Hacia una construcción de ciudadanía ambiental en la escuela. Espiral, Revista de Docencia e Investigación, v.1, n1, p. 13-20. 2011. Recuperado de http://revistas.ustabuca.edu.co/index. php/ESPIRAL/article/view/102/95.

SAUVÉ, L. Perspectivas curriculares para la formación de formadores en educación ambiental. (Ponencia). I Foro Nacional sobre la Incorporación de la PerspectivaAmbientalenlaFormaciónTécnicayProfesional. México. 2004. Recuperado de https://www.miteco.gob.es/ gl/ceneam/articulos-de-opinion/2004_11sauve_tcm37163438.pdf. 
TORRES-RIVERA, A., BADILLO-GAONA, M., VALENTIN-KAJATT, N. O. y RAMÍREZ-MARTÍNEZ, E. T. Las competencias docentes: el desafío de la educación superior. Innovación Educativa, v.14, n.66, p. 129-145. 2014. Recuperado de: http://www.scielo.org.mx/pdf/ie/ v14n66/v14n66a8.pdf.

VALENCIA-MOLINA, T., SERNA-COLLAZOS, A., OCHOA-ANGRINO, S., CAICEDO-TAMAYO. A. M., MONTES-GONZÁLEZ, J. A. y CHÁVEZ-VESCANCE, J. D. Competencias y estándares TIC desde la dimensión pedagógica: Una perspectiva desde los niveles de apropiación de las TIC en la práctica educativa docente. Cali, Colombia: Pontificia Universidad Javeriana. 2016. Recuperado de: http://www.unesco.org/new/fileadmin/ MULTIMEDIA/FIELD/Santiago/pdf/Competenciasestandares-TIC.pdf.

ZAPATA-CALLEJAS, J. El modelo y enfoque de formación por competencias en la Educación Superior: apuntes sobre sus fortalezas y debilidades. Revista Academia y Virtualidad, 8, (2), 24-33. 2015. Recuperado de: https:// dialnet.unirioja.es/descarga/articulo/5236382.pdf.

Recebido em: 29-07-2019 Aprovado em: 29-04-2020 Publicado em: 22-05-2020 